$\vec{w} 2014)$ and have submitted their paper to the European Physical Journal.

"This finding confirms that Hoyle was not a crank," says study co-author Simon Mitton, a science historian at the University of Cambridge, UK, who wrote the 2005 biography Fred Hoyle: A Life in Science. The mere fact that Einstein had toyed with a steady-state model could have lent Hoyle more credibility as he engaged the physics community in a debate on the subject. "If only Hoyle had known, he would certainly have used it to punch his opponents," O'Raifeartaigh says.

Although Hoyle's model was eventually ruled out by astronomical observations, it was at least mathematically consistent, tweaking the equations of Einstein's general theory of relativity to provide a possible mechanism for the spontaneous generation of matter. Einstein's unpublished manuscript suggests that, at first, he believed that such a mechanism could arise

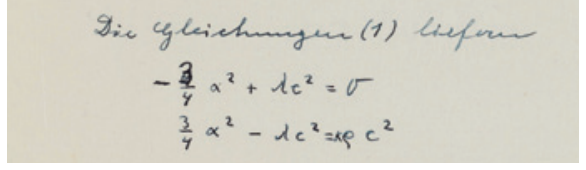

Einstein's correction to his erroneous calculation.

from his original theory without modification. But then he realized that he had made a mistake in his calculations, O'Raifeartaigh and his team suggest. When he corrected it - crossing out a number with a pen of a different colour - he probably decided that the idea would not work and set it aside.

The manuscript was probably "a rough draft commenced with excitement over a neat idea and soon abandoned as the author realized he was fooling himself", says cosmologist James Peebles of Princeton University in New Jersey. There seems to be no record of Einstein ever mentioning these calculations again.
But the fact that Einstein experimented with the steady-state concept demonstrates his continued resistance to the idea of a Big Bang, which he at first found "abominable", even though other theoreticians had shown it to be a natural consequence of his general theory of relativity. (Other leading researchers, such as the eminent Cambridge astronomer Arthur Eddington, were also suspicious of the Big Bang idea, because it suggested a mystical moment of creation.) When astronomers found evidence for cosmic expansion, Einstein had to abandon his bias towards a static Universe, and a steady-state Universe was the next best thing, O'Raifeartaigh and his collaborators say.

Helge Kragh, a science historian at Aarhus University in Denmark, agrees. "What the manuscript shows is that although by then he accepted the expansion of space, [Einstein] was unhappy with a Universe changing in time," he says.

\title{
Fish-kill method questioned
}

\section{Common anaesthetic not the most humane option for zebrafish euthanasia, say studies.}

\section{BY DANIEL CRESSEY}

$\mathrm{R}$ esearchers have called for a common method of killing zebrafish used in laboratories to be abandoned amid growing evidence that it causes unnecessary suffering.

The anaesthetic MS-222, which can be added to tanks to cause overdose, seems to distress the fish, two separate studies have shown. The studies' authors propose that alternative anaesthetics or methods should be used instead.

"These two studies - carried out independently - use different methodologies to reach the same conclusion: zebrafish detect and avoid MS-222 in the water," says Stewart Owen, a senior environmental scientist at AstraZeneca's Brixham Environmental Laboratory in Brixham, UK, and a co-author of one of the studies. "As this is a clear aversive response, as a humane choice, one would no longer use this agent for routine zebrafish anaesthesia."

The use of zebrafish (Danio rerio) in research has skyrocketed in recent years as scientists have sought alternatives to more controversial animal models, such as mammals. The fish are cheap and easy to keep, and although no firm data on numbers have been collected, millions are known to be housed in laboratories around the world. Nearly all will eventually be killed.

MS-222 (ethyl 3-aminobenzoate methanesulphate, also known as TMS) is one of the agents most frequently used to kill the creatures.

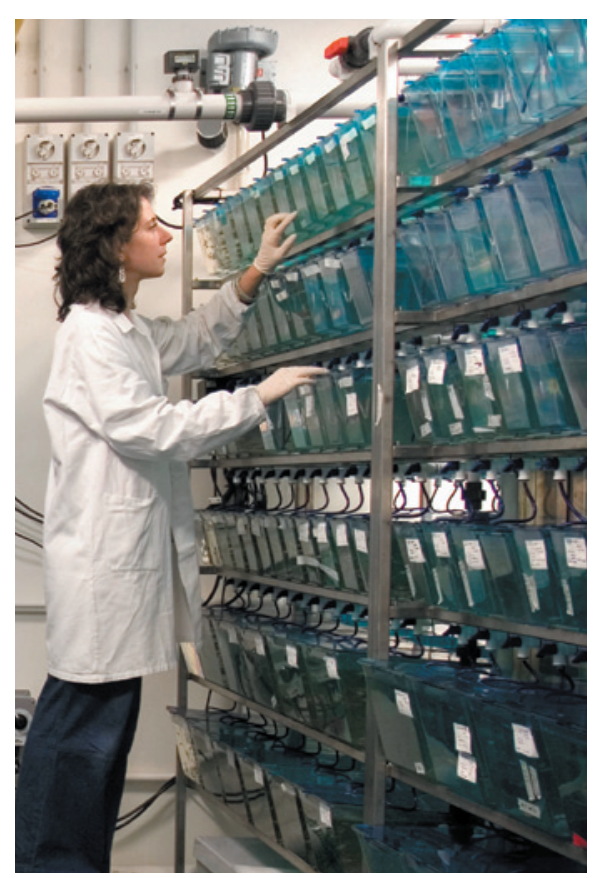

A researcher checks zebrafish tanks in a cancer lab.

It is listed as an acceptable method of euthanasia by many institutions, and also by societies such as the American Veterinary Medical Association. But the study by Owen and his co-authors, published last year (G. D. Readman et al. PLoS ONE 8, e73773; 2013), and the second study, published earlier this month by Daniel Weary and his colleagues at the University of British Columbia in Vancouver, Canada (D. Wong et al. PLoS ONE 9, e88030; 2014), show that zebrafish seem to find the chemical distressing. The research should fundamentally change the practice, say the authors of both papers.

Owen's study used video tracking to measure whether zebrafish avoided anaesthetics flowing through one side of a tank by moving to the other, untreated side. In the case of MS-222, the team found that zebrafish spent significantly more time in the untreated side than on the side containing the anaesthetic.

Weary's team allowed zebrafish to first spend time in either a light or a dark section of a tank, and then exposed them to MS-222 on their preferred side, the light side. After exposure to the anaesthetic, all but one of 17 fish in the study spent less time on the light side, and nine completely avoided it. This indicates that the fish would rather undergo discomfort - in this case, darkness - than be exposed to MS-222.

"There must be something unpleasant" about MS-222 to produce such a strong signal in the experiment, says Weary, because fish do not avoid many other harmful chemicals to such an extent. "The results are pretty clear," he adds. "We're at a stage where it is a matter of getting policy-makers and researchers to think about this and to rethink the procedures."

There is growing debate over the most humane methods of killing laboratory mammals, with rodent euthanasia coming 
- under increased scrutiny (see Nature 500, 130-131; 2013). Fish euthanasia has so far attracted less attention. "I think of fish welfare as being 10 to 20 years behind mammal welfare," says Lynne Sneddon, who studies welfare in fish and is director of bioveterinary science at the University of Liverpool, UK.

Sneddon says the two papers convincingly show that the use of MS-222 to kill zebrafish should probably be avoided. But she notes that there are significant differences between species - data on zebrafish should not be generalized to other laboratory fish, such as salmonids, for example - and therefore cautions against banning its use in the animals entirely.

Zoltan Varga, director of the Zebrafish International Resource Center at the University of Oregon in Eugene, also cautions against abandoning MS-222 because the optimal method of killing will depend heavily on the individual experiment and set-up. "A choice of anaesthetics is critical, as there is a range of reactions possible and we need to administer drugs that address any situation," he says. In some cases, this could be MS-222.

There is not enough evidence to know which is the most humane method, and opinions differ. Owen suggests using the anaesthetic etomidate, which is cheaper than MS-222 (US $\$ 0.15$ per litre compared with $\$ 0.23$ per litre of working solution) and which seemed to be less aversive in his tests. Weary's research suggests clove oil as another cheap alternative. Varga favours 'hypothermal shock' - in which the zebrafish, a tropical species, are rapidly cooled. (This method is illegal in the United Kingdom owing to concerns that ice may damage fish tissue while the animals are still conscious.)

As the number of fish experiments continues to rise - they are the second most popular research species in the United Kingdom - the question grows in importance. "We must have the patience to allow the zebrafish field research time to critically explore what the best - that is, most humane - standards are," says Varga. "We can neither infer these standards from human experience nor from the guidelines and regulations already established for other laboratory organisms." - SEE EDITORIAL P.407

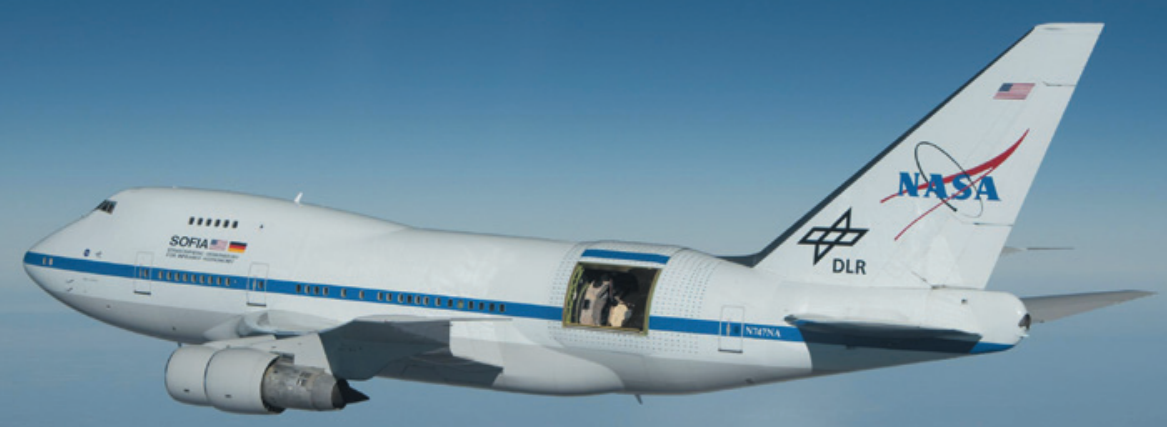

The Stratospheric Observatory for Infrared Astronomy carries a 2.5-metre telescope to viewing hotspots.

ASTRONOMY

SOFIA irons out
technical kinks

US-German airborne telescope slow to reach full capability.

\section{BY ALEXANDRA WITZE}

$\mathrm{L}$ ast October, Diane Wooden was chasing a comet - from the back of a jumbo jet. The aeroplane, known as the Stratospheric Observatory for Infrared Astronomy (SOFIA), is a Boeing 747 modified to hold a 2.5-metre telescope and fly to altitudes of up to 13.7 kilometres, where it escapes most of the atmospheric water vapour that obscures infrared light in the night sky. Wooden, an astrophysicist at the NASA Ames Research Center in Moffett Field, California, was on board in the hope of capturing images of warm dust spewing off Comet ISON (see Nature 506, 281-283; 2014). But soon after she pointed the telescope at the comet, a circuit-breaker failed. It could not be replaced until the plane landed. Instead of nearly an hour observing the comet, Wooden got just a few minutes.

Wooden is still happy with the data she got: the only direct measurement of heat coming off ISON's larger dust grains. But the glitch was typical of those that have plagued the complex flying observatory, a joint project that has so far cost about US $\$ 1.25$ billion for NASA and $\frac{\text { के }}{2}$ the DLR, Germany's aerospace centre.

After years of delays and cost overruns, and many flight hours lost to technical and other issues, SOFIA scientists say that they are finally on the verge of putting their predicaments in the past. On 21 February, a fourth telescope instrument was fully implemented, a long-awaited milestone that means SOFIA has reached full operational capability. "We're entering a different phase now, where our real focus will be on operations and observing and gathering science," says Erick Young, the science mission operations director for SOFIA at Ames. "We'll hit our stride."

Yet just as momentum starts to build, SOFIA will be grounded for a 5.5-month maintenance check mandated by the US government. That will limit the upcoming observing cycle to

\section{TOP STORY}

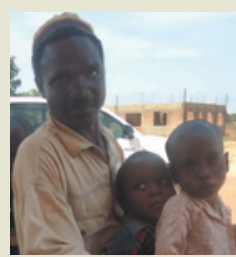

Meningitis immunization campaign in West Africa delivers without ice boxes go.nature.com/1ac7yl

\section{MORE NEWS}

- Wings and fins share a universal geometry across taxa go.nature.com/h26ewg

- Chemists call for boycott over lack of female speakers go.nature.com/3dzkf9 - Publishers withdraw more than 120 gibberish papers go.nature.com/bbtnga

\section{NATURE PODCAST}

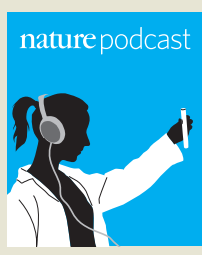

Cancer spread by UV; why Earth's mantle flows; and Einstein's forgotten manuscript nature.com/nature/ podcast 